\title{
Size Ramsey Number of Bounded Degree Graphs for Games
}

\section{Journal Article}

Author(s):

Gebauer, Heidi

Publication date:

2013-07

Permanent link:

https://doi.org/10.3929/ethz-b-000068238

Rights / license:

In Copyright - Non-Commercial Use Permitted

Originally published in:

Combinatorics, Probability \& Computing 22(4), https://doi.org/10.1017/S0963548313000151 


\title{
Size Ramsey Number of Bounded Degree Graphs for Games
}

\author{
HEIDI GEBAUER \\ Institute of Theoretical Computer Science, ETH Zurich, CH-8092 Switzerland \\ (e-mail: gebauerh@inf .ethz.ch)
}

Received 7 August 2012; revised 11 April 2013

\begin{abstract}
We study Maker/Breaker games on the edges of sparse graphs. Maker and Breaker take turns at claiming previously unclaimed edges of a given graph $H$. Maker aims to occupy a given target graph $G$ and Breaker tries to prevent Maker from achieving his goal. We show that for every $d$ there is a constant $c=c(d)$ with the property that for every graph $G$ on $n$ vertices of maximum degree $d$ there is a graph $H$ on at most $c n$ edges such that Maker has a strategy to occupy a copy of $G$ in the game on $H$.

This is a result about a game-theoretic variant of the size Ramsey number. For a given graph $G, \hat{r}^{\prime}(G)$ is defined as the smallest number $M$ for which there exists a graph $H$ with $M$ edges such that Maker has a strategy to occupy a copy of $G$ in the game on $H$. In this language, our result yields that for every connected graph $G$ of constant maximum degree, $\hat{r}^{\prime}(G)=\Theta(n)$.

Moreover, we can also use our method to settle the corresponding extremal number for universal graphs: for a constant $d$ and for the class $\mathcal{G}_{n}$ of $n$-vertex graphs of maximum degree $d, s\left(\mathcal{G}_{n}\right)$ denotes the minimum number such that there exists a graph $H$ with $M$ edges where, for every $G \in \mathcal{G}_{n}$, Maker has a strategy to build a copy of $G$ in the game on $H$. We obtain that $s\left(\mathcal{G}_{n}\right)=\Theta\left(n^{2-\frac{2}{d}}\right)$.
\end{abstract}

2010 Mathematics subject classification: Primary 05C55, 05C57, 91A43, 91A46

\section{Introduction}

Ordinary Ramsey numbers and a game-theoretic variant. The Ramsey number $r(G)$ of a graph $G$ is the smallest number $N$ such that, in any two-colouring of the edges of the complete graph $K_{N}$, there is guaranteed to be a monochromatic copy of $G$. It is well known, for example, that for the complete graph $K_{n}$ on $n$ vertices we have that $2^{\frac{n}{2}} \leqslant r\left(K_{n}\right) \leqslant 4^{n}$, and that, as proved by Chvátal, Rödl, Szemerédi and Trotter [20], for every graph $G$ on $n$ vertices of maximum degree $d$ (the class of graphs we are focusing on in this paper), $r(G) \leqslant c n$, where $c$ depends on $d$ but not on $n$.

A game-theoretic variant of the Ramsey number was introduced by Beck [8]. Two players, called Maker and Breaker, take turns at claiming one previously unclaimed edge 
of $K_{N}$, with Maker going first. Maker's goal is to build a copy of some fixed graph $G$ in his graph and Breaker tries to prevent Maker from achieving his goal: Breaker wins if, after all edges of $K_{N}$ have been claimed, Maker's graph does not contain a copy of $G$. Let $r^{\prime}(G)$ denote the smallest $N$ such that Maker has a strategy to occupy a copy of $G$ in the game on $K_{N}$. A standard strategy-stealing argument shows that $r^{\prime}(G) \leqslant r(G)$. Suppose, for a contradiction, that $r^{\prime}(G)>r(G)$. Thus, for $N:=r(G)$, Breaker has a strategy $S_{B}$ to prevent Maker from building a copy of $G$ in the game on $K_{N}$. Since Maker starts the game he can 'steal' Breaker's strategy by starting with an arbitrary first move and then following $S_{B}$ (if this strategy calls for something he occupied previously, he takes an arbitrary edge: no extra move is disadvantageous for him). The corresponding strategy $S_{M}$ allows Maker to prevent Breaker from occupying a copy of $G$. Suppose that Maker and Breaker play against each other using their respective strategies $S_{M}$ and $S_{B}$ and assume further that Maker colours his edges red and Breaker colours his edges blue. Due to the properties of $S_{M}$ and $S_{B}$, neither player ever occupies a copy of $G$. Hence, as soon as all edges have been claimed, we are given an edge-colouring of $K_{N}$ containing no monochromatic copy of $G$, and therefore $N<r(G)$, contradicting our choice of $N$.

Hence, due to the result of Chvátal, Rödl, Szemerédi and Trotter we immediately obtain that for every graph $G$ of maximum degree $d$ we have that $r^{\prime}(G) \leqslant r(G) \leqslant c(d) n$. Further progress was made when Beck [10] showed that if $N \geqslant \operatorname{poly}(d) 3^{d} \cdot n$, then in the game on $K_{N}$ Maker has a strategy to create a universal graph for the class of graphs on $n$ vertices of maximum degree $d$, i.e., a graph that contains all such graphs ('poly $(d)$ ' denotes a polynomial factor in $d$ ).

Size Ramsey numbers and a game-theoretic variant. The size Ramsey number $\hat{r}(G)$, introduced by Erdős, Faudree, Rousseau and Schelp [21], is the smallest number $M$ for which there exists a graph $H$ with $M$ edges such that any two-colouring of the edges of $H$ yields a monochromatic copy of $G$. Due to a result of Chvátal (see [21]) it is known that $\hat{r}\left(K_{n}\right)=\left(\begin{array}{c}r\left(K_{n}\right) \\ 2\end{array}\right)$, which equals the number of edges in the complete graph on $r\left(K_{n}\right)$ vertices. Naturally, for sparse graphs the situation is quite different. For many sparse graphs $G$ (as cycles and trees of fixed maximum degree) it has been proved that $\hat{r}(G)$ is linear in $n[9,24,25]$. Conversely, Rödl and Szemerédi [28] showed that there exists a graph $G$ on $n$ vertices of maximum degree 3 where

$$
\hat{r}(G) \geqslant c n \log \frac{1}{60} n,
$$

refuting the possibility, raised by Beck and Erdős (see [19]), that for every $d$ there is a constant $c=c(d)$ such that for any graph $G$ on $n$ vertices of maximum degree $d, \hat{r}(G) \leqslant c n$.

The best known upper bound for the class of all graphs with constant maximum degree is due to Kohayakawa, Rödl, Schacht and Szemerédi [26], who derived that for every natural number $d$ there exists a constant $c=c(d)$ such that for every graph $G$ on $n$ vertices of maximum degree $d$,

$$
\hat{r}(G) \leqslant c n^{2-\frac{1}{d}} \log ^{\frac{1}{d}} n .
$$

As for the ordinary Ramsey number, there is a game-theoretic variant of the size Ramsey number: for every graph $G$ we let $\hat{r}^{\prime}(G)$ denote the smallest $M$ for which there 


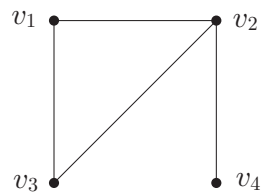

(a)

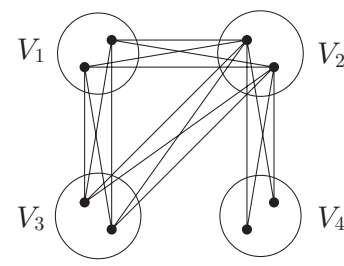

(b)

Figure 1. (a) A graph $G$ and (b) $G^{2}$.

exists a graph $H$ with $M$ edges such that Maker has a strategy to occupy a copy of $G$ in the game on $H$ (by slightly abusing the notion introduced above we also consider games played on non-complete graphs). By a strategy-stealing argument similar to that above, we get that $\hat{r}^{\prime}(G) \leqslant \hat{r}(G)$.

We investigate $\hat{r}^{\prime}(G)$ for graphs $G$ with constant maximum degree $d$. Clearly, $\hat{r}^{\prime}(G) \geqslant$ $|E(G)|$, and thus for connected graphs we have that $\hat{r}^{\prime}(G)$ is at least linear in $n$. Conversely, (1.1) is the best known upper bound (to our knowledge) for $\hat{r}^{\prime}(G)$. We will close this gap by showing that $\hat{r}^{\prime}(G) \leqslant c n$ for some constant $c$ depending on $d$ but not on $n$.

Theorem 1.1. Let $d$ be a natural number. Then there is a constant $c=c(d)$ with the property that for every graph $G$ on $n$ vertices of maximum degree $d$ there is a graph $H$ on at most ch edges such that Maker has a strategy to occupy a copy of $G$ in the game on $H$. In particular,

$$
\hat{r}^{\prime}(G) \leqslant c n .
$$

Let $v_{1}, \ldots, v_{n}$ denote the vertices of $G$ and let $E(G)$ denote the edge set of $G$. The graph $H$ we will construct in the proof of Theorem 1.1 has the additional property that for some carefully chosen constant $c$ (depending on $d$ but not on $n$ ), we will have $H=G^{c}$, where $G^{c}$ denotes the graph obtained by replacing every $v_{i}$ with a set $V_{i}$ of size $c$, and connecting two vertices $u \in V_{i}$ and $v \in V_{j}$ with an edge if and only if $\left(v_{i}, v_{j}\right) \in E(G)$. Figure 1 depicts such a graph. Note that every edge of $G$ corresponds to $c^{2}$ edges of $H$. Thus, $H$ has $c^{2}|E(G)| \leqslant \frac{1}{2} c^{2} d n$ edges. Moreover, the strategy we will describe guarantees that by the end of the game Maker's graph contains a copy of $G$ spanned by some $w_{1} \in V_{1}, \ldots, w_{n} \in V_{n}$, where every $w_{i}$ plays the role of $v_{i}$.

Previous work. Feldheim and Krivelevich [22] considered a related problem. For a given graph $G$ they investigated the minimum number $n_{\text {round }}(G)$ of rounds Maker needs to build a copy of $G$ in the game on a sufficiently large $K_{N}$. Applying a theorem of Alon, Krivelevich, Spencer and Szabó about discrepancy games [6], they derived a strategy for Maker to occupy every given graph $G$ of bounded degree with $n$ vertices in a linear number of rounds (in $n$ ).

Theorem 1.2 (Feldheim and Krivelevich [22]). Let $d$ be an integer. Then there are constants $c=c(d), c^{\prime}=c^{\prime}(d)$ such that for every graph $G$ on $n$ vertices of maximum degree $d$ and every $N>c n$, Maker has a strategy to occupy a copy of $G$ in the game on $K_{N}$ in at most $c^{\prime} n$ rounds. 
We remark that they actually proved this result for the more general class of $d$ degenerate graphs. The values of the constants are $c=d^{11} 2^{2 d+9}$ and $c^{\prime}=d^{11} 2^{2 d+7}$. In our proof of Theorem 1.1 we use some of their findings and approaches, and combine them with several new ideas.

Note that if Maker has a strategy to occupy a copy of $G$ in the game on $H$ then $n_{\text {round }}(G) \leqslant \frac{|E(H)|}{2}$. Hence $\frac{\hat{r}^{\prime}(G)}{2}$ is an upper bound on $n_{\text {round }}(G)$, and thus Theorem 1.1 implies Theorem 1.2 (with weaker constants $c(d), c^{\prime}(d)$ ).

Universal graphs. For a given family $\mathcal{G}$ of graphs, a graph $H$ is called $\mathcal{G}$-universal if $H$ contains a copy of every $G \in \mathcal{G}$. The construction of sparse universal graphs for several families of graphs occurs in the study of VLSI circuit design (see [12] and [18]). Universal graphs for forests, planar graphs, and related classes have been studied in a series of papers (see, e.g., [11, 15, 16, 17, 24], and [7, 11, 13, 14, 27]). Alon, Capalbo, Kohayakawa, Rödl, Ruciński and Szemerédi [4] considered the class $\mathcal{G}_{n, d}$ of graphs on $n$ vertices with maximum degree $d$. They proved that for every $d$ there exists an $\epsilon=\epsilon(d)>0$ such that for every $n$ there is a $\mathcal{G}_{n, d}$-universal graph with at most $n^{2-\epsilon}$ edges. Further progress was made in a series of publications, yielding several deterministic and randomized constructions of sparse $\mathcal{G}_{n, d}$-universal graphs (see, e.g., $[1,2,5]$ ). Finally, Alon and Capalbo [3] found the following.

Theorem 1.3 (Alon and Capalbo [3]). For every $d \geqslant 3$ there exists positive constants $c_{1}, c_{2}$ such that for every $n$ there is an (explicitly constructible) $\mathcal{G}_{n, d}$-universal graph $H$ with at most $c_{1} n$ vertices and at most $c_{2} n^{2-\frac{2}{d}}$ edges.

Theorem 1.3 is tight since it has been shown in [4] that every $\mathcal{G}_{n, d}$-universal graph contains at least $\Omega\left(n^{2-\frac{2}{d}}\right)$ edges. For the remainder of this section, we consider $d$ to be a constant.

Ramsey universal graphs and a game-theoretic variant. For a given family $\mathcal{G}$ of graphs, a graph $H$ is called $\mathcal{G}$-Ramsey-universal if any two-colouring of the edges of $H$ contains a monochromatic $\mathcal{G}$-universal graph. Clearly, the lower bound $\Omega\left(n^{2-\frac{2}{d}}\right)$ (of [4]) on the number of edges in a $\mathcal{G}_{n, d}$-universal graph also serves as a lower bound on the number of edges in a $\mathcal{G}_{n, d}$-Ramsey-universal graph. Conversely, Kohayakawa, Rödl, Schacht and Szemerédi [26] showed that there is a $\mathcal{G}_{n, d}$-Ramsey-universal graph with at most

$$
O\left(n^{2-\frac{1}{d}} \log ^{\frac{1}{d}} n\right)
$$

edges, which is a generalization of (1.1). It is a wide open problem whether this upper bound can be pushed down to $O\left(n^{2-\frac{2}{d}}\right)$.

As for the ordinary Ramsey property, there is a game-theoretic variant of Ramseyuniversality. For a given family $\mathcal{G}$ of graphs, we are interested in those graphs $H$ where for every $G \in \mathcal{G}$, Maker has a strategy to build a copy of $G$ in the game on $H$. (In other words, Maker first fixes $H$ and afterwards Breaker chooses $G$, and then the actual game starts.) We denote the set of these graphs $H$ by $S(\mathcal{G})$ and investigate the smallest number $s=s(\mathcal{G})$ such that there exists a graph $H$ in $S(\mathcal{G})$ with $s$ edges. 
Every graph in $S(\mathcal{G})$ is clearly $\mathcal{G}$-universal. Thus, the lower bound $\Omega\left(n^{2-\frac{2}{d}}\right)$ (of [4]) on the number of edges in a $\mathcal{G}_{n, d}$-universal graph yields that every graph in $S\left(\mathcal{G}_{n, d}\right)$ has at least $\Omega\left(n^{2-\frac{2}{a}}\right)$ edges. Hence,

$$
s\left(\mathcal{G}_{n, d}\right) \geqslant \Omega\left(n^{2-\frac{2}{d}}\right)
$$

Conversely, a standard strategy-stealing argument yields that if a graph $H$ is $G$-Ramsey (i.e., if every two-colouring of the edges of $H$ contains a monochromatic copy of $G$ ) then Maker can build a copy of $G$ in the game on $H$. Thus, every $\mathcal{G}_{n, d}$-Ramsey-universal graph is also in $S\left(\mathcal{G}_{n, d}\right)$. Hence (1.2) implies that some graph in $S\left(\mathcal{G}_{n, d}\right)$ has at most $O\left(n^{2-\frac{1}{d}} \log ^{\frac{1}{d}} n\right)$ edges. Together with (1.3) this gives that

$$
\Omega\left(n^{2-\frac{2}{d}}\right) \leqslant s\left(\mathcal{G}_{n, d}\right) \leqslant O\left(n^{2-\frac{1}{d}} \log ^{\frac{1}{d}} n\right) .
$$

Our construction for proving Theorem 1.1 closes the gap between these two bounds: let $H$ be the graph from Theorem 1.3 and let $H^{\prime}=H^{c}$ for some carefully chosen constant $c$. By construction, $\left|E\left(H^{\prime}\right)\right|=c^{2}|E(H)|=O\left(n^{2-\frac{2}{d}}\right)$, and, furthermore, $H^{\prime}$ contains $G^{c}$ for every $G \in \mathcal{G}_{n, d}$. Thus, by choosing $c$ as in the proof of Theorem 1.1 , for every $G \in \mathcal{G}_{n, d}$, Maker has a strategy to occupy a copy of $G$. Hence, $H^{\prime} \in S\left(\mathcal{G}_{n, d}\right)$ and therefore, $s\left(\mathcal{G}_{n, d}\right) \leqslant O\left(n^{2-\frac{2}{d}}\right)$, which together with $(1.3)$ gives that $s\left(\mathcal{G}_{n, d}\right)=\Theta\left(n^{2-\frac{2}{a}}\right)$.

Notation. We first define some game-theoretic notions. Following the standard notation, for a graph property $\mathcal{P}$ of $N$-vertex graphs and a graph $H$ on the vertex set $V(H)=V\left(K_{N}\right)$, we let $(E(H), \mathcal{P})$ denote the game where Maker's goal is to create a graph which possesses $\mathcal{P}$. In this paper we investigate the case where $\mathcal{P}$ is the property that the graph contains a copy of a fixed graph $G$. We call $H$ the base graph or the board. The base graph along with the sets of Maker's and Breaker's claimed edges is called a game position, or just a position for short. Adopting the notation of [22], to distinguish between vertices of $G$ and vertices of $H$ we mark the vertices of $H$ with an asterisk.

Throughout this paper we will assume that Breaker starts the game. Otherwise Maker can start with an arbitrary move, then follow his strategy. If his strategy calls for an edge he already claimed he takes an arbitrary edge (he can only benefit from extra moves). By slightly modifying the standard notation, we let a round denote a pair consisting of a Breaker's move and the consecutive Maker's move.

We will also need some graph terminology. Let $G$ be a graph and let $u, v \in V(G)$. The neighbourhood $N_{G}(v)$ of $v$ denotes the set of vertices which are adjacent to $v$ in $G$. The distance $\operatorname{dist}_{G}(u, v)$ between $u$ and $v$ is defined as the number of edges in a shortest path in $G$ connecting $u$ and $v$. For a fixed ordering $v_{1}, \ldots, v_{n}$ of the vertices of $G$ we let $N_{G}^{-}\left(v_{i}\right)=N_{G}\left(v_{i}\right) \cap\left\{v_{1}, \ldots, v_{i-1}\right\}$, and $N_{G}^{+}\left(v_{i}\right)=N_{G}\left(v_{i}\right) \cap\left\{v_{i+1}, \ldots, v_{n}\right\}$. For $U \subseteq V(G), E_{G}(U)$ denotes the set of edges spanned by $U$ and, similarly, for disjoint subsets $U, W \subseteq V(G)$, $E_{G}(U, W)$ denotes the set of edges with one endpoint in $U$ and the other in $W$. When there is no danger of confusion we sometimes omit the index $G$. Furthermore, $G \backslash U$ denotes the graph obtained by taking $G$ and deleting all the vertices of $U$. The subgraph induced by $U, G[U]$, denotes the graph obtained by deleting all vertices of $V(G) \backslash U$ in $G$.

Let $D$ be a directed acyclic graph and let $u, v \in V(D)$. If $(u, v) \in E(D)$ then $v$ is called an out-neighbour of $u$. If there is a directed path of length at least one from $u$ to $v$ in $D$, 
then $v$ is called a descendant of $u$. In other words, the descendants of $u$ are exactly the out-neighbours of $u$ in the transitive closure of $D$. Note that according to this convention, no vertex is considered a descendant of itself.

Ceiling and floor signs are routinely omitted whenever they are not crucial for clarity.

Organization of this paper. In Section 2 we give rough descriptions of the method of Feldheim and Krivelevich [22], and our contribution. Moreover, we also illustrate the main structural differences between the respective problems.

A key ingredient of our approach is a carefully specified ordering $\Pi$ of the vertices of $G$. In Section 3 we define $\Pi$ and point out some of its properties, which will be essential for Maker's strategy.

In Sections 4 and 5 we give criteria motivated by [22], and explain how Maker can make use of them in certain subgames of our strategy. In Section 6 we finally develop an overall strategy $S$ for Maker and derive that, following $S$, Maker will eventually win the game.

\section{Sketch of a known result and our contribution}

Let $G$ be a graph of maximum degree $d$ and suppose that $V(G)=\left\{v_{1}, \ldots, v_{n}\right\}$. We first give a rough sketch of Maker's strategy of [22] for constructing $G$ fast in the game on $K_{N}$. Maker processes the vertices $v_{1}, \ldots, v_{n}$ one by one. After processing $v_{1}, \ldots, v_{i-1}$ he chooses a set $W_{i} \subseteq V\left(K_{N}\right)$ of 'fresh vertices' (i.e., vertices where no incident edge has been claimed by Maker or Breaker) where $\left|W_{i}\right|$ is some fixed constant (depending on $d$ but not on $n$ ). Then he starts claiming edges connecting $W_{i}$ with $W_{1}, \ldots, W_{i-1}$, according to an involved sub-strategy, such that Maker's graph obtains the following property. For every index set $i_{1}<\cdots<i_{k}<i$ and every common neighbour $v_{j}$ of $v_{i_{1}}, \ldots, v_{i_{k}}, v_{i}$ in $\left\{v_{1}, \ldots, v_{i_{1}-1}\right\}$, it holds that for every choice $w_{i_{1}}^{\star}, \ldots, w_{i_{k}}^{\star}, w_{i}^{\star}$ of respective vertices in $W_{i_{1}}, \ldots, W_{i_{k}}, W_{i}$ there are relatively many common neighbours in $W_{j}$. Naturally, this sub-strategy strongly depends on the actual outcome of processing $v_{1}, \ldots, v_{i-1}$.

Feldheim and Krivelevich derived that their strategy guarantees that by the end of the game Maker can find vertices $w_{1}^{\star} \in W_{1}, w_{2}^{\star} \in W_{2}, \ldots, w_{n}^{\star} \in W_{n}$ such that $\left\{w_{1}^{\star}, \ldots, w_{n}^{\star}\right\}$ span a copy of $G$, where every $w_{i}^{\star}$ plays the role of $v_{i}$.

In our construction for proving Theorem 1.1 we want to economize the number of edges of the base graph. To this end we choose a suitable constant $c$ and replace $K_{N}$ with $H:=G^{c}$. Recall that $G^{c}$ denotes the graph obtained by replacing every $v_{i}$ with a set $V_{i}$ of size $c$, and connecting two vertices $u \in V_{i}$ and $v \in V_{j}$ with an edge if and only if $\left(v_{i}, v_{j}\right) \in E(G)$. Moreover, we set $W_{i}:=V_{i}$ for every $i \in\{1, \ldots, n\}$. So, in contrast to the above strategy, the $W_{i}$ are already determined at the beginning of the game. The method of processing $v_{1}, \ldots v_{n}$ one by one does not carry over to the new situation, since Breaker could easily occupy all edges incident to $W_{n}$ before Maker finished processing $v_{1}, \ldots, v_{n-1}$.

The main ingredient of our approach is a carefully chosen ordering of the vertices combined with a modified strategy for Maker with the property that for every $i$, Maker can start processing $v_{i}$ after processing only a certain small subset $T_{i} \subseteq\left\{v_{1}, \ldots, v_{i-1}\right\}$. We represent this relation by a directed graph $D$ on the vertex set $\left\{v_{1}, \ldots, v_{n}\right\}$, where we add 


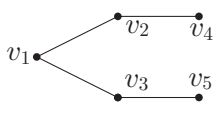

(a)

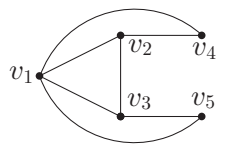

(b)

Figure 2. (a) A graph $G$ and (b) $D_{2}(G)$.

an edge from $v_{i}$ to every vertex in $T_{i}$. We extend Maker's strategy as follows. Whenever Breaker claims an edge in $E\left(W_{1} \cup \cdots \cup W_{i-1}, W_{i}\right)$ before all vertices in $T_{i}$ have been processed, then Maker continues processing a descendant of $v_{i}$ in $D$. We will derive (using some structural properties of $D$ ) that this approach allows Maker to eventually occupy a copy of $G$.

\section{Defining a suitable ordering of the vertices}

Let $G$ be a graph. Using the standard notation, $\Delta(G)$ denotes the maximum degree of $G$ and the chromatic number $\chi(G)$ denotes the minimum $c$ such that $G$ is $c$-colourable. We will apply the following well-known bound.

Lemma 3.1. For every graph $G, \chi(G) \leqslant \Delta(G)+1$.

This can be seen as follows. Let $G=(V, E)$, let $V=\left\{v_{1}, \ldots, v_{n}\right\}$ and let

$$
C=\{1, \ldots, \Delta(G)+1\}
$$

be a set of colours. By colouring the vertices in the order $v_{1}, \ldots, v_{n}$, assigning to $v_{i}$ the smallest colour of $C$ not already used on a neighbour of $v_{i}$, we obtain a proper colouring.

For a given graph $G=(V, E)$, we let $D_{2}(G)=\left(V^{\prime}, E^{\prime}\right)$ denote the graph where $V^{\prime}=V$ and $E^{\prime}$ consists of all pairs $(u, v)$ of vertices where $u \neq v$ and $u, v$ have distance at most two in $G$. Figure 2 shows an example. Note that, with $\Delta:=\Delta(G)$, the maximum degree of $D_{2}(G)$ is at most $\Delta+\Delta(\Delta-1)=\Delta^{2}$. Moreover, every independent set of $D_{2}(G)$ corresponds to a subset $S \subseteq V$ where every two vertices in $S$ have distance at least three in $G$. The next corollary is a direct consequence of Lemma 3.1 .

Corollary 3.2. Let $G=(V, E)$ be a graph and let $\Delta=\Delta(G)$. Then $\chi\left(D_{2}(G)\right) \leqslant \Delta^{2}+1$. In particular, we can colour $V$ with colours $\left\{1, \ldots, \Delta^{2}+1\right\}$ such that every two vertices of the same colour have distance at least 3 in $G$.

Colouring the vertices. From now on we let $G$ be a fixed graph on $n$ vertices of maximum degree $d$ and we let $l: V(G) \rightarrow\left\{1, \ldots, d^{2}+1\right\}$ be the colouring of Corollary 3.2. Figure 4(a) shows an example. We also fix an ordering $v_{1}, \ldots, v_{n}$ of the vertices in $V(G)$ such that $l\left(v_{1}\right) \leqslant l\left(v_{2}\right) \leqslant \cdots \leqslant l\left(v_{n}\right)$. Finally, we assume without loss of generality that $d \geqslant 2$. (Note that if the maximum degree is at most 1 then we can just consider a supergraph $G^{\prime} \supseteq G$ with maximum degree 2.) 


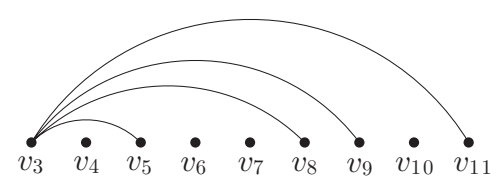

Figure 3. An example for $i=3$ and $j=11$. We have $S_{3,11}=\{5,8,9\}$.

Let $1 \leqslant i<j \leqslant n$. We say that an index $r$ is neighbourwise sandwiched between $i$ and $j$ if $i<r<j$ and $v_{r} \in N_{G}\left(v_{i}\right)$. For every $\left(v_{i}, v_{j}\right) \in E(G)$ where $i<j$, we let $S_{i, j}$ denote the set of indices $r$ which are neighbourwise sandwiched between $i$ and $j$. Figure 3 shows an illustration. Moreover, for every index $j$ we let $\mathcal{S}_{j}$ denote the union of all sets $S_{i}:=\{i\} \cup S_{i, j}$ where $v_{i} \in N_{G}^{-}\left(v_{j}\right)$. Note that all vertices in $\mathcal{S}_{j}$ have distance at most two from $v_{j}$, thus

$$
\left|\mathcal{S}_{j}\right| \leqslant d+d(d-1)=d^{2} .
$$

It will turn out that for every $j$ the set of indices $\mathcal{S}_{j}$ play an important role in Maker's strategy. Intuitively, it will be the case that he can only process a vertex $v_{j}$ as soon as he has finished processing all vertices $v_{i}$ where $i \in \mathcal{S}_{j}$ (more details will be given below).

For further discussion we aim to express the relation ' $i \in \mathcal{S}_{j}$ ' with a graph. Let $D$ denote the directed graph on the vertex set $v_{1}, \ldots, v_{n}$ where there is an arc from $v_{j}$ to $v_{i}$ if and only if $i \in \mathcal{S}_{j}$. Figures $4(\mathrm{~b})$ and $4(\mathrm{c})$ depict an example. For every arc $\left(v_{k}, v_{i}\right) \in E(D)$ we have $l\left(v_{k}\right) \geqslant l\left(v_{i}\right)$ and $l\left(v_{k}\right) \neq l\left(v_{i}\right)$ (because $\left.\operatorname{dist}\left(v_{i}, v_{k}\right) \leqslant 2\right)$, thus $l\left(v_{k}\right)>l\left(v_{i}\right)$, and therefore $D$ is acyclic.

Observation 3.3. For every $j \in\{1, \ldots, n\}$ we have that the number of descendants of $v_{j}$ in $D$ is at most $\left(d^{2}\right)^{d^{2}+1}$.

This can be seen as follows. By (3.1) and the construction of $D$, every vertex has at most $d^{2}$ out-neighbours in $D$. Since $l\left(v_{k}\right)>l\left(v_{i}\right)$ for every arc $\left(v_{k}, v_{i}\right)$ in $D$, the vertices of every directed path in $D$ have distinct colours. Thus, every directed path in $D$ with start vertex $v_{j}$ has at most $l\left(v_{j}\right) \leqslant d^{2}+1$ vertices. So the number of descendants of $v_{j}$ is at most

$$
\sum_{i=1}^{l\left(v_{j}\right)-1}\left(d^{2}\right)^{i} \leqslant\left(d^{2}\right)^{l\left(v_{j}\right)} \leqslant\left(d^{2}\right)^{d^{2}+1}
$$

(here we use the assumption that $d \geqslant 2$ ).

In terms of the intuition formulated above, Observation 3.3 yields that in order to be able to process a vertex $v_{j}$, Maker only has to complete the processing of a constant number of vertices. A precise analysis will be given in Section 6 when we devise Maker's overall strategy.

Construction of the board. Recall that we fixed an ordering of the vertices $v_{1}, \ldots, v_{n}$ of $G$ according to the colouring $l$ of Corollary 3.2. Let

$$
\begin{aligned}
c_{d} & =d^{5} 2^{d+4}, \quad \text { and } \\
c & =d c_{d}^{2} d^{2 d^{2}+2}+c_{d}+2 .
\end{aligned}
$$




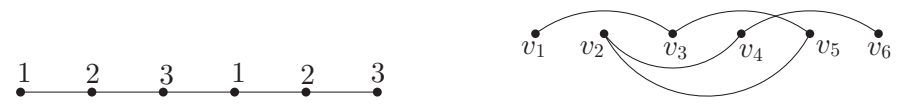

(a)

(b)

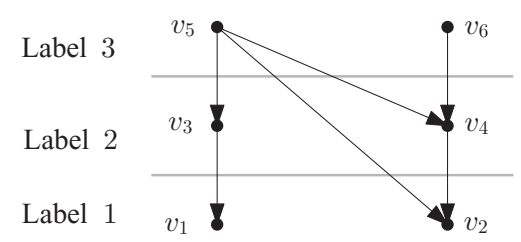

(c)

Figure 4. (a) A colouring $l$ of a path of length 5 such that vertices with the same colour have distance at least 3 , and (b) an ordering of the vertices according to $l$. Figure (c) shows the corresponding graph $D$.

We set

$$
H:=G^{c} .
$$

Recall that, by definition of $G^{c}, H$ is the graph resulting from replacement of every $v_{i}$ with a set $V_{i}$ of size $c$ and connecting two vertices $u \in V_{i}$ and $v \in V_{j}$ with an edge if and only if $\left(v_{i}, v_{j}\right) \in E(G)$.

\section{Candidates and candidate schemes}

We introduce the concepts of a candidate vertex and a candidate scheme by stating adapted versions of Definitions 2.1-2.3 in [22]. Recall that, according to (3.4), $V(H)=V_{1} \cup \cdots \cup V_{n}$, and that a position is the base graph $H$ along with the sets of Maker's and Breaker's claimed edges. The intuition behind the $B_{k} \subseteq V_{k}$ used in the next definitions is that during the game, as part of his strategy, Maker will define for every $k \in\{1, \ldots, n\}$ an appropriate subset $B_{k} \subseteq V_{k}$ with $\left|B_{k}\right|=c_{d}$.

Definition 4.1 (vertex candidate with respect to a specific edge). Let $H^{\star}$ be a position, let $\left(v_{i}, v_{j}\right) \in E(G)$ where $i<j$, and suppose that $S_{i, j}=\left\{k_{1}, \ldots, k_{t-1}\right\}$. Moreover, for every $k \in\{i\} \cup\left\{k_{1}, \ldots, k_{t-1}\right\}$, let $B_{k} \subseteq V_{k}$ be a non-empty set. A vertex $x^{\star} \in V_{j}$ is called a candidate with respect to the edge $\left(v_{i}, v_{j}\right)$ and the family $\mathcal{B}=\left\{B_{i}, B_{k_{1}}, \ldots, B_{k_{t-1}}\right\}$ if, for every choice of vertices $b_{1}^{\star} \in B_{k_{1}}, b_{2}^{\star} \in B_{k_{2}}, \ldots, b_{t-1}^{\star} \in B_{k_{t-1}}$, we have

$$
\frac{\mid\left\{b^{\star} \in B_{i}: \text { Maker claimed }\left(b^{\star}, b_{1}^{\star}\right), \ldots,\left(b^{\star}, b_{t-1}^{\star}\right),\left(b^{\star}, x^{\star}\right) \text { in } H^{\star}\right\} \mid}{\left|B_{i}\right|} \geqslant \frac{1}{t 2^{t}} \text {. }
$$

Figure 5 illustrates Definition 4.1. Note that if no index is neighbourwise sandwiched between $i$ and $j$ (i.e., if $t=1$ ) then a vertex $x^{\star} \in V_{j}$ is a candidate with respect to $\left(v_{i}, v_{j}\right)$ and $\mathcal{B}=\left\{B_{i}\right\}$ if and only if $x^{\star}$ is connected to at least half of the vertices of $B_{i}$ in Maker's graph. 


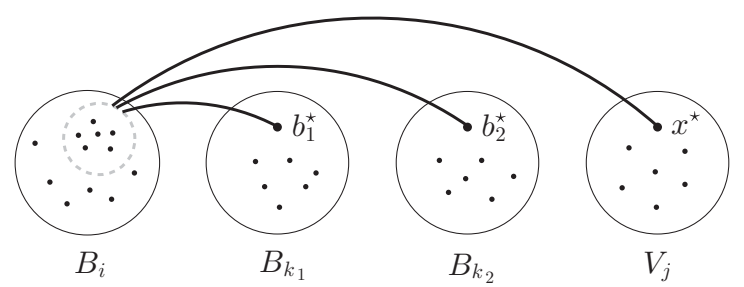

Figure 5. An illustration of Definition 4.1 for $t=3$. Many vertices in $B_{i}$ are connected to all three vertices $b_{1}^{\star}, b_{2}^{\star}, x^{\star}$.

We will now specify those vertices $x^{\star} \in V_{j}$ which are candidates for every edge $\left(v_{i}, v_{j}\right) \in$ $E(G)$ with $i<j$. Recall that $\mathcal{S}_{j}$ denotes the union of all sets $S_{i}:=\{i\} \cup S_{i, j}$ where $v_{i} \in$ $N_{G}^{-}\left(v_{j}\right)$.

Definition 4.2 (vertex candidate). Let $H^{\star}$ be a position, let $1 \leqslant j \leqslant n$ and for every $k \in \mathcal{S}_{j}$ let $B_{k} \subseteq V_{k}$. Moreover, let $\mathcal{B}=\left\{B_{k}: k \in \mathcal{S}_{j}\right\}$. A vertex $x^{\star} \in V_{j}$ is called a candidate with respect to the family $\mathcal{B}$ if, for every $v_{i} \in N_{G}^{-}\left(v_{j}\right), x^{\star}$ is a candidate with respect to $\left(v_{i}, v_{j}\right)$ and $\left\{B_{i}\right\} \cup\left\{B_{k}: k \in S_{i, j}\right\}$.

Note that if $N_{G}^{-}\left(v_{j}\right)=\emptyset$ then every $x^{\star} \in V_{j}$ is a candidate with respect to the empty set. Finally, we define a candidate scheme which, as we will see, guarantees that Maker's graph contains a copy of $G$.

Definition 4.3 (candidate scheme). Let $H^{\star}$ be a position, and for every $1 \leqslant j \leqslant n$, let $B_{j} \subseteq V_{j}$ with $\left|B_{j}\right| \geqslant d 2^{d}$. We say that $\left(B_{1}, B_{2}, \ldots, B_{n}\right)$ form a candidate scheme if, for every $1 \leqslant j \leqslant n$ and every $x^{\star} \in B_{j}, x^{\star}$ is a candidate with respect to $\left\{B_{k}: k \in \mathcal{S}_{j}\right\}$.

The next lemma, which is a slight adaptation of Lemma 2.1 in [22], shows that a candidate scheme is sufficient for Maker's win.

Lemma 4.4 (Feldheim and Krivelevich [22]). Let $H^{\star}$ be a position and let $\left(B_{1}, B_{2}, \ldots, B_{n}\right)$ be a candidate scheme. Then Maker's graph contains a copy of $G$.

For completeness we reproduce the proof given in [22].

Proof of Lemma 4.4. Our goal is to construct an embedding $\Phi:\left\{v_{1}, \ldots, v_{n}\right\} \rightarrow V(H)$ such that $\Phi\left(v_{i}\right) \in B_{i}$ for every $1 \leqslant i \leqslant n$, and $\Phi\left(v_{1}\right), \ldots, \Phi\left(v_{n}\right)$ span a copy of $G$ in Maker's graph. We proceed inductively, starting from $\Phi\left(v_{n}\right)$ and moving down to $\Phi\left(v_{1}\right)$. First, we choose an arbitrary $x^{\star} \in B_{n}$ and set $\Phi\left(v_{n}\right):=x^{\star}$. Let $i \leqslant n-1$ and suppose that we have already defined $\Phi\left(v_{i+1}\right), \ldots, \Phi\left(v_{n}\right)$ in such a way that they span a copy of $G\left[\left\{v_{i+1}, \ldots, v_{n}\right\}\right]$ in Maker's graph. If $N_{G}^{+}\left(v_{i}\right)=\emptyset$ then we choose an arbitrary vertex $x^{\star} \in B_{i}$ as $\Phi\left(v_{i}\right)$ : clearly, $\Phi\left(v_{i}\right), \ldots, \Phi\left(v_{n}\right)$ span a copy of $G\left[\left\{v_{i}, \ldots, v_{n}\right\}\right]$ in Maker's graph. Otherwise, let $\left\{v_{k_{1}}, \ldots, v_{k_{t}}\right\}=N_{G}^{+}\left(v_{i}\right)$ and note that by assumption, $t \leqslant d$. We suppose without loss of generality that $k_{1}<k_{2}<\cdots<k_{t}$. Since $\Phi\left(v_{k_{t}}\right)$ is a candidate with respect to the edge 
$\left(v_{i}, v_{k_{t}}\right)$ and $\left\{B_{i}, B_{k_{1}}, \ldots, B_{k_{t-1}}\right\}$, we get that

$$
\mid\left\{b^{\star} \in B_{i}: \text { Maker claimed }\left(b^{\star}, \Phi\left(v_{k_{1}}\right)\right), \ldots,\left(b^{\star}, \Phi\left(v_{k_{t}}\right)\right) \text { in } H^{\star}\right\} \mid \geqslant \frac{\left|B_{i}\right|}{t 2^{t}},
$$

which is at least $\left|B_{i}\right| /\left(d 2^{d}\right) \geqslant 1$. We choose one of these vertices to be $\Phi\left(v_{i}\right)$. This implies that $\Phi\left(v_{i}\right), \ldots, \Phi\left(v_{n}\right)$ span a copy of $G\left[\left\{v_{i}, \ldots, v_{n}\right\}\right]$ in Maker's graph.

\section{Two suitable subgames}

Our goal is to devise a strategy which allows Maker to obtain a candidate scheme. To this end we first analyse two appropriate subgames. We will need the concept of untouched vertices. Let $H^{\star}$ be a position. A vertex $x^{\star} \in V_{j}$ is touched if Maker or Breaker has claimed an edge of the form $\left(y^{\star}, x^{\star}\right)$ where $y^{\star} \in V_{1} \cup V_{2} \cup \cdots \cup V_{j-1}$. Otherwise $x^{\star}$ is called untouched.

In the first subgame Maker's goal is to achieve the situation described in Definition 4.1.

Definition 5.1. Let $H^{\star}$ be a position, let $\left(v_{i}, v_{j}\right) \in E(G)$ where $i<j$, and suppose that $S_{i, j}=\left\{k_{1}, \ldots, k_{t-1}\right\}$. Moreover, for every $k \in\{i\} \cup\left\{k_{1}, \ldots, k_{t-1}\right\}$, let $B_{k} \subseteq V_{k}$ be a nonempty set. For every vertex $x^{\star} \in V_{j}$ which is untouched in $H^{\star}$, the game $G_{B_{i}, x^{\star}}$ with respect to $\left\{B_{k_{1}}, \ldots, B_{k_{t-1}}\right\}$ is defined as follows. The board consists of the set of edges in $E_{H}\left(B_{i},\left\{x^{\star}\right\}\right)$, and Maker's goal is to achieve that $x^{\star}$ becomes a candidate with respect to $\left(v_{i}, v_{j}\right)$ and $\left\{B_{i}, B_{k_{1}}, \ldots, B_{k_{t-1}}\right\}$.

Note that for some positions $H^{\star}$, the game is hopeless for Maker; e.g., for every $H^{\star}$ where for some $1 \leqslant r \leqslant t-1$ and some $y^{\star} \in B_{k_{r}}$, all edges in $E_{H}\left(B_{i},\left\{y^{\star}\right\}\right)$ belong to Breaker's graph. However, the overall strategy for Maker, which we will describe, guarantees that the $B_{k}$ are convenient.

Maker's goal in the second subgame is that several $x^{\star} \in V_{j}$ become a candidate with respect to all edges $\left(v_{i}, v_{j}\right)$ where $i<j$.

Definition 5.2. Let $H^{\star}$ be a position, let $1 \leqslant j \leqslant n$, and for every $k \in \mathcal{S}_{j}$, let $B_{k} \subseteq V_{k}$ be a non-empty set. For every set $B_{j} \subseteq V_{j}$ where all vertices of $B_{j}$ are untouched in $H^{\star}$, the game $G_{B_{j}}$ with respect to $\left\{B_{k}: k \in \mathcal{S}_{j}\right\}$ is defined as follows. The board consists of the union of the edge-sets $E_{H}\left(B_{i}, B_{j}\right)$ where $v_{i} \in N_{G}^{-}\left(v_{j}\right)$, and Maker's goal is to achieve that every $x^{\star} \in B_{j}$ becomes a candidate with respect to $\left\{B_{k}: k \in \mathcal{S}_{j}\right\}$.

We will show that Maker has a strategy to determine the $B_{j}$ in such a way that he succeeds in every $G_{B_{j}}$, which finally allows him to obtain a candidate scheme.

We first express the size of the board of the game $G_{B_{j}}$ in terms of the sizes of the $B_{i}$ where $v_{i} \in N_{G}^{-}\left(v_{j}\right)$.

Observation 5.3. Let $H^{\star}$ be a position, let $1 \leqslant j \leqslant n$, and for every $k \in \mathcal{S}_{j} \cup\{j\}$ let $B_{k} \subseteq V_{k}$ be as in Definition 5.2. The size of the board of the game $G_{B_{j}}$ (with respect to $\left\{B_{k}: k \in \mathcal{S}_{j}\right\}$ ) is $\sum_{v_{i} \in N_{G}^{-}\left(v_{j}\right)}\left|B_{i}\right|\left|B_{j}\right|$. 
We now use an adaptation of Lemma 2.2 in [22] to show that for an appropriate choice of the $B_{k_{1}}, \ldots, B_{k_{t-1}}$, Maker has a strategy to win the game $G_{B_{i}, x^{\star}}$ described in Definition 5.1. Recall that in (3.2) we set $c_{d}:=d^{5} 2^{d+4}$.

Lemma 5.4. Let $H^{\star}$ be a given position, let $\left(v_{i}, v_{j}\right) \in E(G)$ where $i<j$, and suppose that $S_{i, j}=\left\{k_{1}, \ldots, k_{t-1}\right\}$. Moreover, for every $k \in\{i\} \cup\left\{k_{1}, \ldots, k_{t-1}\right\}$, let $B_{k} \subseteq V_{k}$ where $\left|B_{k}\right|=c_{d}$. Suppose that for every $1 \leqslant r \leqslant t-1$ we have that every $y^{\star} \in B_{k_{r}}$ is a candidate with respect to $\left(v_{i}, v_{k_{r}}\right)$ and $\left\{B_{i}\right\} \cup\left\{B_{k_{1}}, \ldots, B_{k_{r-1}}\right\}$. Then, for every $x^{\star} \in V_{j}$ which is untouched in $H^{\star}$, Maker has a strategy to win $G_{B_{i}, x^{\star}}$ with respect to $\left\{B_{k_{1}}, \ldots, B_{k_{t-1}}\right\}$.

As usual, a hypergraph $F$ is a pair $(V, E)$, where $V$ is a finite set whose elements are called vertices and $E$ is a family of subsets of $V$, called hyperedges. We consider the game where Maker and Breaker alternately claim an unclaimed vertex of $V$ until all vertices are claimed. We will use the following result by Alon, Krivelevich, Spencer and Szabó [6], extending a previous result by Székely [29].

Theorem 5.5 (Alon, Krivelevich, Spencer and Szabó [6]). Let $F$ be a hypergraph with $X$ hyperedges, whose smallest hyperedge contains at least $x$ vertices. Then Maker has a strategy to claim at least

$$
\frac{x}{2}-\sqrt{\frac{x \ln (2 X)}{2}}
$$

vertices of each hyperedge.

Proof of Lemma 5.4. The proof follows the same lines as the proof of Lemma 2.2 in [22]. If $t=1$ then Maker can win easily: in each of his moves he claims a free edge connecting $x^{\star}$ with a vertex in $B_{i}$; this guarantees that by the end of the game half of the edges in $E\left(B_{i},\left\{x^{\star}\right\}\right)$ belong to Maker's graph, as claimed. Suppose now that $t \geqslant 2$ and let $F$ denote the hypergraph where $V(F)=E\left(B_{i},\left\{x^{\star}\right\}\right)$, and $E(F)$ is obtained by adding (to the initially empty set), for every choice of vertices $b_{1}^{\star} \in B_{k_{1}}, b_{2}^{\star} \in B_{k_{2}}, \ldots, b_{t-1}^{\star} \in B_{k_{t-1}}$, the hyperedge

$$
e_{b_{1}^{\star}, \ldots, b_{t-1}^{\star}}:=\left\{\left(b^{\star}, x^{\star}\right) \in E\left(B_{i},\left\{x^{\star}\right\}\right): \text { Maker claimed }\left(b^{\star}, b_{1}^{\star}\right), \ldots,\left(b^{\star}, b_{t-1}^{\star}\right)\right\} .
$$

By interpreting $G_{B_{i}, x^{\star}}$ as a game on $F$, we get that Lemma 5.4 is equivalent to the statement that Maker has a strategy to claim at least

$$
\frac{\left|B_{i}\right|}{t 2^{t}}=\frac{c_{d}}{t 2^{t}}
$$

vertices of each hyperedge. Our goal is to show the latter. By (3.2) we have that

$$
|E(F)| \leqslant \prod_{r=1}^{t-1}\left|B_{k_{r}}\right|=c_{d}^{t-1}=\left(d^{5} 2^{d+4}\right)^{t-1} .
$$

Let $b_{1}^{\star} \in B_{k_{1}}, \ldots, b_{t-1}^{\star} \in B_{k_{t-1}}$ be any choice of vertices. We get that $\left|e_{b_{1}^{\star}, \ldots, b_{t-1}^{\star}}\right|=\mid\left\{b^{\star} \in\right.$ $B_{i}$ : Maker claimed $\left.\left(b^{\star}, b_{1}^{\star}\right), \ldots,\left(b^{\star}, b_{t-1}^{\star}\right)\right\} \mid$. Since (by assumption) $b_{t-1}^{\star}$ is a candidate with 
respect to $\left(v_{i}, v_{k_{t-1}}\right)$ and $\left\{B_{i}, B_{k_{1}}, \ldots, B_{k_{t-2}}\right\}$, we obtain that

$$
\left|e_{b_{1}^{\star}, \ldots, b_{t-1}^{\star}}\right| \geqslant \frac{\left|B_{i}\right|}{(t-1) 2^{t-1}}=\frac{c_{d}}{(t-1) 2^{t-1}}=\frac{d^{5} 2^{d+4}}{(t-1) 2^{t-1}} .
$$

By Theorem 5.5, Maker has a strategy to claim at least

$$
\frac{d^{5} 2^{d+4}}{(t-1) 2^{t}}-\sqrt{\frac{d^{5} 2^{d+4}}{(t-1) 2^{t}} \ln \left(2\left(d^{5} 2^{d+4}\right)^{t-1}\right)}
$$

vertices of each hyperedge. Further calculations show that the expression in (5.1) is at least

$$
\frac{d^{5} 2^{d-t+4}}{t}=\frac{c_{d}}{t 2^{t}}
$$

(Details can be found in [22].) This concludes the proof.

The next corollary is a consequence of Lemma 5.4.

Corollary 5.6. Let $H^{\star}$ be a position, let $1 \leqslant j \leqslant n$, and for every $k \in \mathcal{S}_{j}$, let $B_{k} \subseteq V_{k}$ where $\left|B_{k}\right|=c_{d}$. Suppose that for every $v_{i} \in N_{G}^{-}\left(v_{j}\right)$ and every $q \in S_{i, j}$ we have that every $y^{\star} \in B_{q}$ is a candidate with respect to $\left(v_{i}, v_{q}\right)$ and $\left\{B_{i}\right\} \cup\left\{B_{r}: r \in S_{i, q}\right\}$. Then, for every $B_{j} \subseteq V_{j}$ where all vertices of $B_{j}$ are untouched in $H^{\star}$, Maker has a strategy to win $G_{B_{j}}$ with respect to $\left\{B_{k}: k \in \mathcal{S}_{j}\right\}$.

Proof. We first note that for every $v_{i} \in N_{G}^{-}\left(v_{j}\right)$ the conditions of Lemma 5.4 are satisfied for the edge $\left(v_{i}, v_{j}\right)$ and the family $\left\{B_{k}: k \in\{i\} \cup S_{i, j}\right\}$. Indeed, let $S_{i, j}=\left\{k_{1}, \ldots, k_{t-1}\right\}$. By assumption, for every $k_{r} \in\left\{k_{1}, \ldots, k_{t-1}\right\}$ we have that all vertices in $B_{k_{r}}$ are candidates with respect to $\left(v_{i}, v_{k_{r}}\right)$ and $\left\{B_{i}\right\} \cup\left\{B_{s}: s \in S_{i, k_{r}}\right\}=\left\{B_{i}\right\} \cup\left\{B_{k_{1}}, \ldots, B_{k_{r-1}}\right\}$.

Consider the following strategy for Maker. Suppose that Breaker claims an edge $\left(b^{\star}, x^{\star}\right)$ with $b^{\star} \in B_{i}$ and $x^{\star} \in B_{j}$ where $i<j$. Then Maker responds in the game $G_{B_{i}, x^{\star}}$ (with respect to $\left\{B_{r}: r \in S_{i, j}\right\}$ ).

Since the boards of the games $G_{B_{i}, x^{\star}}$ are pairwise disjoint, Maker can treat each game $G_{B_{i}, x^{\star}}$ separately. Thus Lemma 5.4 yields a winning strategy for Maker in $G_{B_{j}}$.

The next observation shows that the game $G_{B_{j}}$ is finished after a reasonably small number of rounds.

Observation 5.7. Let $H^{\star}$ be a position, let $1 \leqslant j \leqslant n$, and for every $k \in \mathcal{S}_{j} \cup\{j\}$ let $B_{k} \subseteq V_{k}$ be such that the conditions of Corollary 5.6 are satisfied. Suppose also that $\left|B_{j}\right|=c_{d}$. By Observation 5.3 the size of the board of the game $G_{B_{j}}$ (with respect to $\left\{B_{k}: k \in \mathcal{S}_{j}\right\}$ ) is $\sum_{v_{i} \in N_{G}^{-}\left(v_{j}\right)}\left|B_{i}\right|\left|B_{j}\right| \leqslant d c_{d}^{2}$, and hence $G_{B_{j}}$ lasts at most $d c_{d}^{2}$ rounds.

\section{Obtaining a candidate scheme via the subgames}

Equipped with Corollary 5.6 we can now describe a strategy for Maker to obtain a candidate scheme (which due to Lemma 4.4 guarantees that Maker's graph contains a 


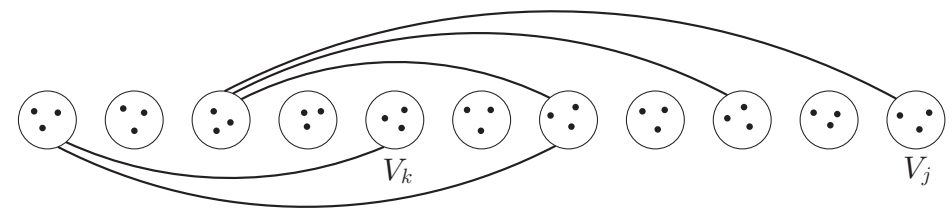

Figure 6. An example of an index $k$ with $v_{k} \in \operatorname{Desc}\left(v_{j}\right)$. (A thick line indicates that the two corresponding vertex sets form a complete bipartite graph.)

copy of the target graph $G)$. We first need some more notation. Recall that $D$ denotes the directed graph where $V(D)=\left\{v_{1}, \ldots, v_{n}\right\}$ and $E(D)=\left\{\left(v_{j}, v_{i}\right): i \in \mathcal{S}_{j}\right\}$. For every vertex $v_{j}$ we let $N_{\text {out }}\left(v_{j}\right)$ denote the set of out-neighbours of $v_{j}$ and we let $\operatorname{Desc}\left(v_{j}\right)$ denote the set of descendants of $v_{j}$. (Figure 6 depicts one element of $\operatorname{Desc}\left(v_{j}\right)$.)

During the game Maker will determine for each $j \in\{1, \ldots, n\}$ a subset $B_{j} \subseteq V_{j}$ with $\left|B_{j}\right|=c_{d}$ whose vertices are all untouched at the time of its determination. For a vertex $v_{j}$ where for every $k \in \mathcal{S}_{j} \cup\{j\}$ the set $B_{k}$ has already been determined, we say that $v_{j}$ is completed if every $x^{\star} \in B_{j}$ is a candidate with respect to $\left\{B_{k}: k \in \mathcal{S}_{j}\right\}$. Note that, as soon as $v_{1}, \ldots, v_{n}$ are all completed, $\left(B_{1}, \ldots, B_{n}\right)$ form a candidate scheme, and thus Maker won.

Furthermore, we call a vertex $v_{j}$ ready if every vertex $v_{k}$ where $k \in \mathcal{S}_{j}$ is completed. In other words, $v_{j}$ is ready if all vertices in $N_{\text {out }}\left(v_{j}\right)$ are completed. As part of his strategy, for every $1 \leqslant j \leqslant n$, Maker determines $B_{j}$ as soon as $v_{j}$ becomes ready. In the following, for every ready vertex $v_{j}$ we abbreviate by ' $G_{B_{j}}$ ' the game $G_{B_{j}}$ with respect to $\left\{B_{k}: k \in \mathcal{S}_{j}\right\}$ (note that since $v_{j}$ is ready the $B_{k}$ are all determined).

Maker's strategy will have the property that every completed vertex is also ready. Sometimes we want to find a vertex which is ready but not completed.

Observation 6.1. Let $H^{\star}$ be a position and let $v_{j}$ be a non-completed vertex. Then there is at least one vertex $v_{k} \in\left\{v_{j}\right\} \cup \operatorname{Desc}\left(v_{j}\right)$ which is ready but not completed.

This can be seen as follows. Let $k$ be the smallest index such that $v_{k}$ is a noncompleted vertex in $\left\{v_{j}\right\} \cup \operatorname{Desc}\left(v_{j}\right)$. Every $v_{l} \in N_{\text {out }}\left(v_{k}\right)$ has the property that $l<k$, hence by minimality all vertices in $N_{\text {out }}\left(v_{k}\right)$ are completed, thus $v_{k}$ is ready.

Maker's strategy. At the very beginning of the game, for every $v_{j}$ where $\mathcal{S}_{j}=\emptyset$, Maker chooses $B_{j}$ to be an arbitrary subset of $V_{j}$ where $\left|B_{j}\right|=c_{d}$. (Thus, by definition, $v_{j}$ is completed.) By slightly abusing notation, we refer to this as round zero. For every vertex $v_{i}$ which became ready in round zero, Maker also determines an arbitrary $B_{i} \subseteq V_{i}$ of size $c_{d}$. Maker will proceed in such a way that after each round $r$ the following two invariants are maintained.

(I1) For every vertex $v_{j}$ which is not yet ready, at least $c_{d}+2$ vertices of $V_{j}$ are untouched. (I2) Let $v_{j}$ be a vertex which is ready but not completed. Then there is at least one unclaimed edge in the board of the game $G_{B_{j}}$.

At the very beginning of the game (i.e., after round 0) the invariants (I1) and (I2) clearly hold. Suppose that $r-1$ rounds have been played. By induction we can assume that (I1) and (I2) are fulfilled so far. If all vertices $v_{1}, \ldots, v_{n}$ are completed then Maker stops 
playing (because he won). Otherwise the game continues. (I2) together with Observation 6.1 guarantees that there are still unoccupied edges. Assume that in round $r$ Breaker claims the edge $\left(x^{\star}, y^{\star}\right)$, and suppose that $x^{\star} \in V_{i}, y^{\star} \in V_{j}$ and $i<j$. We distinguish three cases.

Case 1: $v_{j}$ is not ready. Then Observation 6.1 guarantees that there is at least one vertex $v_{k} \in \operatorname{Desc}\left(v_{j}\right)$ such that $v_{k}$ is ready but not completed. Maker claims an arbitrary free edge in the board of $G_{B_{k}}$. (The existence of such an edge is guaranteed by (I2).)

Case 2: $v_{j}$ is ready but not completed. Then Maker responds in the game $G_{B_{j}}$.

Case 3: $v_{j}$ is completed. Then Maker selects some vertex $v_{k}$ which is ready but not completed (such a vertex can be obtained by selecting a non-completed vertex and applying Observation 6.1), and claims an arbitrary free edge in the board of $G_{B_{k}}$.

In any case, if a vertex $v_{k}$ became ready in round $r$ then Maker chooses a subset $S \subseteq V_{k}$ of $c_{d}+2$ vertices which were all untouched after round $r-1$ (such an $S$ exists due to (I1)). Note that at most two vertices $z_{1}^{\star}, z_{2}^{\star} \in S$ became touched in round $r$. Maker sets $B_{k}:=S \backslash\left\{z_{1}^{\star}, z_{2}^{\star}\right\}$.

Checking the invariants. We first show that (I1) is satisfied after round $r$. To this end we fix a vertex $v_{j}$ which is non-ready after round $r$. Note that due to his strategy Maker has not claimed any edge incident to a vertex in $V_{j}$ so far. We observe that every time a vertex $y^{\star} \in V_{j}$ was touched by Breaker, Maker selected some (ready) vertex $v_{k} \in \operatorname{Desc}\left(v_{j}\right)$ and occupied an edge in the board of $G_{B_{k}}$ (here it is crucial that a vertex $y^{\star} \in V_{j}$ is only called touched if some player claimed an edge in $E\left(V_{1} \cup \cdots \cup V_{j-1},\left\{y^{\star}\right\}\right)$ ). We set $f\left(y^{\star}\right):=v_{k}$ (if $y^{\star}$ was touched several times then we just choose one $v_{k}$ with this property). Then the number of touched vertices in $V_{j}$ (after $r$ rounds) is bounded by the number of pre-images of $\operatorname{Desc}\left(v_{j}\right)$ under $f$. We have

$$
\left|f^{-1}\left(\operatorname{Desc}\left(v_{j}\right)\right)\right|=\left|\cup_{v_{k} \in \operatorname{Desc}\left(v_{j}\right)} f^{-1}\left(\left\{v_{k}\right\}\right)\right| .
$$

By Observation 5.3, the board size of every $G_{B_{k}}$ is at most $d c_{d}^{2}$, and thus

$$
\left|f^{-1}\left(\left\{v_{k}\right\}\right)\right| \leqslant d c_{d}^{2}
$$

Hence, by Observation 3.3 we obtain that

$$
\left|f^{-1}\left(\operatorname{Desc}\left(v_{j}\right)\right)\right| \leqslant d c_{d}^{2}\left|\operatorname{Desc}\left(v_{j}\right)\right| \leqslant d c_{d}^{2} d^{2 d^{2}+2},
$$

which together with (3.3) and (3.4) implies that at least $c_{d}+2$ vertices in $V_{j}$ remain untouched. Hence (I1) is fulfilled after round $r$.

We now show (I2). Let $v_{j}$ be a vertex which is ready after round $r$, and assume that $v_{j}$ became ready in round $r^{\prime} \leqslant r$. It can be checked that (due to Maker's strategy and the definition of readiness) directly after round $r^{\prime}$ the conditions of Corollary 5.6 were satisfied for $v_{j}$. Note that Maker's strategy has the property that from this point on, whenever Breaker claims an edge of the board of $G_{B_{j}}$, Maker will respond in $G_{B_{j}}$. In this way Maker can treat each such game separately, following the strategy of Corollary 5.6. This guarantees that Maker will eventually win $G_{B_{j}}$, implying that $v_{j}$ will become completed. So, as soon as all edges of the board of $G_{B_{j}}$ are occupied, $v_{j}$ is completed. 
Proof of Maker's win. We first note that due to (I1), (I2) and Observation 6.1, Maker can always make a move according to his strategy unless all vertices $v_{1}, \ldots, v_{n}$ are completed (thus he won). Since the board is finite, the latter will eventually occur. This concludes the proof of Theorem 1.1.

\section{Open problems}

Degenerate graphs. The famous Burr-Erdős conjecture says that for every $d$ there is a constant $c=c(d)$ such that every $d$-degenerate graph $G$ with $n$ vertices satisfies that $r(G) \leqslant c n$. (Recall that $r(G)$ denotes the ordinary Ramsey number, i.e., the smallest number $N$ such that, in any two-colouring of the edges of the complete graph $K_{N}$, there is guaranteed to be a monochromatic copy of G.) Chvátal, Rödl, Szemerédi, and Trotter [20] settled it for the class of graphs of maximum degree $d$. In its full generality the conjecture, however, is still open. The best known upper bound for the class of all $d$-degenerate graphs $H$, due to Fox and Sudakov [23], is $r(H) \leqslant 2^{c(d) \sqrt{\log n} n}$.

For the game-theoretic Ramsey number $\hat{r}^{\prime}(G)$ the situation is similar: Feldheim and Krivelevich showed that Maker can build a candidate scheme for every ordering $v_{1}, \ldots, v_{n}$ of the vertices where every vertex $v_{i}$ has at most $d$ neighbours in $\left\{v_{i+1}, \ldots, v_{n}\right\}$. Hence Theorem 1.2 also holds for $d$-degenerate graphs.

For our proof of Theorem 1.1, which does not only economize the number of rounds but also the number of edges of the base graph, the situation is different. It is crucial that, in addition, every vertex $v_{i}$ has at most $d$ neighbours in $\left\{v_{1}, \ldots, v_{i-1}\right\}$ : this allows us to determine an ordering of the vertices where the sets $\mathcal{S}_{j}$ are all small. For $d \geqslant 2$ it remains open whether Theorem 1.1 can be generalized to the class of $d$-degenerate graphs.

Open Problem 7.1. Is it true that for every $d \geqslant 2$ there is a constant $c=c(d)$ such that $\hat{r}^{\prime}(G) \leqslant c n$ for every d-degenerate graph on $n$ vertices?

For 1-degenerate graphs $G$ (i.e., forests) $\hat{r}^{\prime}(G)$ is at most linear in $n$, which follows from a straightforward modification of the proof in [22]. For a direct proof, let $H:=G^{2}$ (recall that $G^{2}$ denotes the graph obtained by replacing every $v_{i}$ with a set $V_{i}$ of size 2 , and connecting two vertices $u \in V_{i}$ and $v \in V_{j}$ with an edge if and only if $\left(v_{i}, v_{j}\right) \in E(G)$ ), and consider the following strategy of Maker. Whenever Breaker claims an edge $\left(x^{\star}, y^{\star}\right)$ where, say, $x^{\star} \in V_{i}, y^{\star} \in V_{j}$ and $i<j$, then Maker occupies the remaining edge in $E\left(V_{i},\left\{y^{\star}\right\}\right)$. This allows Maker to obtain a candidate scheme, which guarantees that by the end of the game his graph contains a copy of $G$.

To our knowledge, Open Problem 7.1 remains unsolved even if we restrict to the case $d=2$.

\section{Acknowledgement}

We would like to thank Tibor Szabó for many helpful discussions on the topic of this paper. 


\section{References}

[1] Alon, N. and Asodi, V. (2002) Sparse universal graphs. J. Comput. Appl. Math. 142 1-11.

[2] Alon, N. and Capalbo, M. (2007) Sparse universal graphs for bounded degree graphs. Random Struct. Alg. 31 123-133.

[3] Alon, N. and Capalbo, M. (2008) Optimal universal graphs with deterministic embedding. In Proc. 19th Annual ACM-SIAM Symposium on Discrete Algorithms (SODA), pp. 373378.

[4] Alon, N., Capalbo, M., Kohayakawa, Y., Rödl, V., Ruciński, A. and Szemerédi, E. (2000) Universality and tolerance. In Proc. 41st IEEE Symposium on Foundations of Computer Science (FOCS), pp. 14-21.

[5] Alon, N., Capalbo, M., Kohayakawa, Y., Rödl, V., Ruciński, A. and Szemerédi, E. (2001) Near-optimum universal graphs for graphs with bounded degrees. In RANDOM-APPROX, pp. $170-180$.

[6] Alon, N., Krivelevich, M., Spencer, J. and Szabó, T. (2005) Discrepancy games. Electron. J. Combin. 12 R51.

[7] Babai, L., Chung, F. R. K., Erdős, P., Graham, R. L. and Spencer, J. (1982) On graphs which contain all sparse graphs. Ann. Discrete Math. 12 21-26.

[8] Beck, J. (1981) Van der Waerden and Ramsey type games. Combinatorica 1 103-116.

[9] Beck, J. (1983) On size Ramsey number of paths, trees and cycles I. J. Graph Theory 7 $115-130$.

[10] Beck, J. (1994) Deterministic graph games and a probabilistic intuition. Combin. Probab. Comput. 3 13-26.

[11] Bhatt, S. N., Chung, F., Leighton, F. T. and Rosenberg, A. (1989) Universal graphs for boundeddegree trees and planar graphs. SIAM J. Discrete Math. 2 145-155.

[12] Bhatt, S. N. and Leiserson, C. E. (1984) How to assemble tree machines. In Advances in Computing Research 2, (F. P. Preparata, ed.) JAI Press, Greenwich, CT, pp. 95-114.

[13] Capalbo, M. (1999) A small universal graph for bounded-degree planar graphs. In Proc. 10th Annual ACM-SIAM Symposium on Discrete Algorithms (SODA), pp. 150-154.

[14] Capalbo, M. and Kosaraju, S. R. (1999) Small universal graphs. In Proc. 31st Annual ACM Symposium on Theory of Computing (STOC), pp. 741-749.

[15] Chung, F. R. K. and Graham, R. L. (1978) On graphs which contain all small trees. J. Combin. Theory Ser. B 24 14-23.

[16] Chung, F. R. K. and Graham, R. L. (1979) On universal graphs. Ann. New York Acad. Sci. 319 136-140.

[17] Chung, F. R. K. and Graham, R. L. (1983) On universal graphs for spanning trees. Proc. London Math. Soc. 27 203-211.

[18] Chung, F. R. K., Rosenberg, A. L. and Snyder, L. (1983) Perfect storage representations for families of data structures. SIAM J. Alg. Discrete Methods 4 548-565.

[19] Chung, F. and Graham, R. (1998) Erdős on Graphs: His Legacy of Unsolved Problems, A. K. Peters.

[20] Chvátal, V., Rödl, V., Szemerédi, E. and Trotter, W. T. (1983) The Ramsey number of a graph with bounded maximum degree. J. Combin. Theory Ser. B 34 239-243.

[21] Erdős, P., Faudree, R. J., Rousseau, C. C. and Schelp, R. H. (1978) The size Ramsey number. Period. Math. Hungar. 9 145-161.

[22] Feldheim, O. N. and Krivelevich, M. (2008) Winning fast in sparse graph construction games. Combin. Probab. Comput. 17 781-791.

[23] Fox, J. and Sudakov, B. (2009) Two remarks on the Burr-Erdőos conjecture. Europ. J. Combin. 30 1630-1645.

[24] Friedman, J. and Pippenger, N. (1987) Expanding graphs contain all small trees. Combinatorica 7 71-76. 
[25] Haxell, P. E., Kohayakawa, Y. and Łuczak, T. (1995) The induced size-Ramsey number of cycles. Combin. Probab. Comput. 4 217-239.

[26] Kohayakawa, Y., Rödl, V., Schacht, M. and Szemerédi, E. (2011) Sparse partition universal graphs for graphs of bounded degree. Adv. Math. 226 5041-5065.

[27] Rödl, V. (1981) A note on universal graphs. Ars Combin. 11 225-229.

[28] Rödl, V. and Szemerédi, E. (2000) On size Ramsey numbers of graphs with bounded degree. Combinatorica 20 257-262.

[29] Székely, L. A. (1984) On two concepts of discrepancy in a class of combinatorial games. In Infinite and Finite Sets, Vol. 37 of Colloquia Mathematica Societatis Janos Bolyai, pp. 679-683. 